\title{
ESCALA DE AVALIAÇÃO DOS AMBIENTES DA PRÁTICA PROFISSIONAL DE ENFERMAGEM: CONSTRUÇÃO E VALIDAÇÃO DE CONTEÚDO
}

\author{
SCALE OF EVALUATION OF THE ENVIRONMENTS OF \\ PROFESSIONAL NURSING PRACTICE: CONSTRUCTION \\ AND CONTENT VALIDATION
}

\section{ESCALA DE EVALUACIÓN DE LOS AMBIENTES DE LA PRÁCTICA PROFESIONAL DE ENFERMERÍA: CONSTRUCCIÓN Y VALIDACIÓN DE CONTENIDO}

\author{
Olga Maria Pimenta Lopes Ribeiro ${ }^{1}$ \\ Corália Maria Fortuna de Brito Vicente ${ }^{2}$ \\ Maria Manuela Ferreira Pereira da Silva Martins ${ }^{3}$ \\ Letícia de Lima Trindade ${ }^{4}$ \\ Clemente Neves de Sousa ${ }^{5}$ \\ Maria Filomena Passos Teixeira Cardoso
}

Como citar este artigo: Ribeiro OMPL, Vicente CMFB, Martins MMFPS, Trindade LL, Sousa CN, Cardoso MFPT. Escala de avaliação dos ambientes da prática profissional de enfermagem: construção e validação de conteúdo. Rev baiana enferm. 2020;34:e37996.

Objetivo: construir e validar o conteúdo da Escala de Avaliação dos Ambientes da Prática Profissional de Enfermagem. Método: estudo metodológico realizado de janeiro a maio de 2020. A construção da Escala foi antecedida de pesquisa qualitativa prévia e de revisão de literatura. A validação de conteúdo foi efetuada por 22 peritos. Resultados: inicialmente a Escala tinha 128 itens agrupados nas dimensões estrutura, processo e resultado. Decorrente da avaliação dos peritos, na estrutura dos 65 itens iniciais, foram excluídos 20, reformulados 10 e adicionado um. No processo, dos 49 itens iniciais, excluíram-se 8 e reformularam-se 2 . No resultado, dos 14 itens iniciais, foram excluídos 2, reformulados 2 e adicionado 1. A versão final ficou com 100 itens, cujo Índice de Validade de Conteúdo de cada item oscilou entre 0,86 e 1 . Conclusão: a construção e posterior validação dos itens pelos peritos foi uma etapa fundamental, dando segurança à continuidade dos procedimentos psicométricos.

Descritores: Estudos de Validação. Ambiente de Trabalho. Prática Profissional. Enfermagem. Garantia da Qualidade dos Cuidados de Saúde.

\footnotetext{
Enfermeira. Doutora em Ciências da Enfermagem. Professora Adjunta na Escola Superior de Enfermagem do Porto. Porto, Portugal. olgaribeiro@esenf.pt. http://orcid.org/0000-000 I-9982-9537.

Doutora em Ciências Biomédicas. Professora Catedrática no Instituto de Ciências Biomédicas Abel Salazar da Universidade do Porto. Porto, Portugal. http://orcid. org/0000-0002-2082-4483.

3 Enfermeira. Doutora em Ciências da Enfermagem. Professora Coordenadora na Escola Superior de Enfermagem do Porto. Porto, Portugal. http://orcid.org/00000003-1527-9940.

Enfermeira. Doutora em Enfermagem. Professora na Universidade do Estado de Santa Catarina e na Universidade Comunitária da Região de Chapecó. Chapecó, Santa Catarina, Brasil. http://orcid.org/0000-0002-7I 19-0230.

Enfermeiro. Doutor em Ciências da Enfermagem. Professor Adjunto na Escola Superior de Enfermagem do Porto. Porto, Portugal. http://orcid.org/0000-0003-26540497.

Enfermeira Gestora no Centro Hospitalar Universitário de São João. Professora na Universidade Fernando Pessoa. Porto, Portugal. http://orcid.org/0000-000 I-57582310.
} 
Objective: to construct and validate the content of the Assessment Scale of Nursing Professional Practice Environments. Method: methodological study conducted from January to May 2020. The construction of the Scale after a previous qualitative research and literature review. Content validation was performed by 22 experts. Results: initially, the Scale had 128 items grouped in the dimensions structure, process and outcome. Due to the experts' evaluation, in the structure dimension, the 65 initial items, 20 were excluded, 10 were reformulated and one, added. In the process dimension, of the 49 initial items, 8 were excluded and 2 were reformulated. In the outcome dimension, of the 14 initial items, 2 were excluded, 2 reformulated and added 1. The final version contained 100 items, in which the Content Validity Index of each item fluctuated between 0.86 and 1. Conclusion: the construction and subsequent validation of the items by the experts was a fundamental step, giving security to the continuity of psychometric procedures.

Descriptors: Validation Studies. Working Environment. Professional Practice. Nursing. Quality Assurance, Health Care.

Objtivo: construir y validar el contenido de la Escala de Evaluación de Ambientes de Práctica Profesional de Enfermería. Método: estudio metodológico realizado de enero a mayo de 2020. La construcción de la Escala ocurrió después de investigaciones cualitativas previas y revisión de la literatura. La validación del contenido fue realizada por 22 expertos. Resultados: inicialmente, la Escala tenía 128 elementos agrupados en las dimensiones estructura, proceso y resultado. Debido a la evaluación de los expertos, en la dimensión estructura, de los 65 puntos iniciales, se excluyeron 20, 10 fueron reformulados y uno añadido. En la dimensión proceso, de los 49 puntos iniciales, se excluyeron 8 y se reformularon 2. En la dimensión resultado, de los 14 elementos iniciales, 2 fueron excluidos, 2 reformulados y añadidos 1. La versión final fue de 100 elementos, cuyo Índice de Validez de Contenido de cada elemento fluctuó entre 0,86 y 1. Conclusión: la construcción y posterior validación de los elementos por parte de los expertos fue un paso fundamental, dando seguridad a la continuidad de los procedimientos psicométricos.

Descriptores: Estudios de validación. Ambiente de Trabajo. Práctica Profesional. Enfermería. Garantía de la Calidad de Atención de Salud.

\section{Introdução}

Os enfermeiros e o ambiente onde trabalham têm um papel fundamental na segurança do paciente, bem como na qualidade dos cuidados prestados $^{(1)}$. Em estudos recentes ${ }^{(2-3)}$, confirmou-se que existem fatores preditivos comuns à qualidade e à segurança do paciente, entre eles a satisfação no trabalho e as restrições organizacionais. Estas sofrem influências de recursos insuficientes e da falta de apoio dos órgãos de gestão. $\mathrm{Na}$ perspetiva dos autores, o investimento nessas áreas culminará numa melhoria da qualidade e da segurança dos cuidados prestados ${ }^{(2-3)}$.

Apesar de ser objeto de estudo desde os anos 1980, o ambiente da prática profissional em enfermagem, definido como o conjunto de características do contexto de trabalho que a facilitam ou constrangem ${ }^{(4)}$, tem merecido especial atenção nos últimos anos ${ }^{(3,5-6)}$. Se, por um lado, na sequência do experienciado pelos profissionais de enfermagem e da exigência cada vez maior dos pacientes, a necessidade de melhorar as condições de trabalho possa ter incrementado investimentos nos ambientes da prática, por outro, tem-se tornado urgente clarificar porque motivo, nos contextos da prática, nem sempre é notória a evolução da profissão, nomeadamente perante o significativo desenvolvimento da disciplina de Enfermagem ${ }^{(7)}$. Neste contexto, embora o envolvimento dos enfermeiros no desempenho do seu exercício profissional seja importante, é crucial identificar como as instituições têm criado condições que garantam ambientes favoráveis à qualidade dos cuidados.

A literatura evidencia que ambientes da prática profissional de enfermagem favoráveis são caracterizados por recursos materiais adequados, quantitativo de pessoal suficiente, liderança e suporte aos enfermeiros, boas relações profissionais, participação efetiva dos enfermeiros nas políticas internas da organização e investimento em fundamentos de enfermagem que garantam a qualidade dos cuidados ${ }^{(4-5,8)}$.

Investigações sustentam que um ambiente da prática profissional de enfermagem favorável 
contribui para a satisfação dos enfermeiros, para menores taxas de Burnout, para a sua retenção nas organizações, para a redução dos custos, para a otimização dos resultados em relação aos clientes, culminando globalmente na melhoria da qualidade dos cuidados em enfermagem ${ }^{(3,5,9-10)}$. No contexto internacional, os Magnet Hospitals têm sido reconhecidos como aqueles que apresentam ambientes da prática profissional mais favoráveis ${ }^{(5)}$, já que evidenciam um conjunto de características com potencial para atrair e reter os enfermeiros, de que são exemplo, o reconhecimento da autonomia, a tomada de decisão partilhada, a responsabilização pela qualidade dos cuidados prestados, a gestão e liderança efetivas, a adequação de pessoal e a flexibilidade de horários.

A própria World Health Organization, no relatório sobre o estado da Enfermagem no Mundo, relembra a necessidade de os diversos países proporcionarem um ambiente favorável à prática de enfermagem, de modo a atrair, reter e motivar a força de trabalho em Enfermagem, verdadeiramente insubstituível para a saúde global das populações ${ }^{(11)}$. Além de serem os principais intervenientes na prestação direta de cuidados, os enfermeiros são também determinantes na avaliação da qualidade e segurança dos cuidados prestados aos clientes, sejam eles doentes, famílias e/ou cuidadores ${ }^{(2)}$.

Neste contexto, atendendo à relevância dos ambientes da prática profissional para a garantia da qualidade dos cuidados em enfermagem e, simultaneamente, para o bem-estar dos enfermeiros, a sua avaliação é necessária para conhecer-se as fragilidades e propor-se estratégias que melhorem a sua qualidade. Tanto no contexto internacional como no nacional têm sido frequentemente usados como instrumentos o Practice Environment Scale of the Work Nursing Index ${ }^{(4)}$ e o Revised Nursing Work Index ${ }^{(12)}$. Embora apresentem dimensões e itens diferentes, ambos permitem avaliar a percepção dos enfermeiros quanto à presença de um conjunto de características organizacionais no ambiente hospitalar. Como confirmado em revisão de literatura $^{(13)}$, o fato de estarem essencialmente focados nas condições estruturais da organização, pode ser considerado um limite desses instrumentos, bem como sua adaptação à realidade norteamericana e a falta de adequação ao contexto atual.

Ainda cabe ponderar que, embora esta temática seja profundamente estudada no contexto internacional, são escassas as investigações em Portugal sobre os ambientes da prática profissional em enfermagem, bem como sobre a falta de instrumentos adequados às especificidades dos contextos de trabalho, às particularidades do próprio exercício profissional de enfermagem praticado no país, e que, simultaneamente, contemplem todos os componentes fundamentais à qualidade dos cuidados de enfermagem, isto é, a estrutura, o processo e o resultado ${ }^{(14)}$.

Em recente revisão de literatura $^{(13)}{ }$ identificaram-se dez instrumentos para avaliar os ambientes da prática profissional de enfermagem. Em uns, prevaleceu o foco na estrutura, enquanto em outros, destacou-se particularmente o processo. Embora um desses instrumentos já esteja validado para a população portuguesa ${ }^{(15-16)}$ e outros possam ser adaptados transculturalmente à realidade do país, não seria possível, com recurso de um único instrumento, avaliar todos os componentes dos ambientes da prática profissional determinantes para a qualidade da assistência em enfermagem.

Nesse contexto, tornou-se premente o desenvolvimento de um instrumento mais abrangente. O modelo proposto por Donabedian ${ }^{(14)}$, usado na concepção desse instrumento, tradicionalmente permite considerar os diferentes componentes da qualidade, sendo a estrutura, o processo e o resultado, os três elementos determinantes na avaliação dos ambientes da prática profissional em enfermagem favoráveis à qualidade dos cuidados. O autor, considerado pioneiro nos estudos sobre este último aspecto, com olhar direcionado ao contexto hospitalar, é um clássico nos estudos de qualidade em saúde.

Decorrente do mencionado, este estudo tem como objetivo construir e validar o conteúdo da Escala de Avaliação dos Ambientes da Prática Profissional de Enfermagem. 


\section{Método}

Estudo metodológico que apresenta a construção e validação de conteúdo de um instrumento para avaliação dos ambientes da prática profissional em enfermagem. Inicialmente, para a identificação dos itens a serem incluídos no instrumento, foram analisados os dados de uma pesquisa qualitativa prévia realizada com a participação de 56 enfermeiros de 19 instituições hospitalares das cinco Regiões de Saúde de Portugal continental ${ }^{(17)}$. Nessa investigação de natureza fenomenológica, com a participação de enfermeiros de cuidados gerais, enfermeiros especialistas e enfermeiros gestores, foram identificados os fatores dos ambientes da prática profissional de enfermagem que podem promover ou comprometer a qualidade dos cuidados de enfermagem no contexto hospitalar. Importa referir que esses fatores corresponderam a atributos perfeitamente integrados na tríade de Donabedian: estrutura, processo e resultado ${ }^{(14)}$. Posteriormente, com a realização de uma revisão de literatura ${ }^{(13)}$, além de terem sido identificados, no contexto internacional, dez instrumentos que avaliavam os ambientes da prática profissional de enfermagem, confirmou-se a pertinência de desenvolver um instrumento mais integrador, tendo sido também possível melhorar a redação e evitar a redundância entre os itens incluídos no instrumento a ser submetido a avaliação.

Após essa etapa, da análise realizada dos itens a incluir no instrumento, tornou-se relevante alicerçar a concepção da Escala em três dimensões: estrutura, processo e resultado. Assim, na sequência dos achados nas entrevistas realizadas no âmbito do estudo qualitativo prévio ${ }^{(17)}$ e resultante da revisão da literatura ${ }^{(13)}$, a primeira versão da Escala de Avaliação dos Ambientes da Prática Profissional de Enfermagem (EAAPPE) ficou constituída por 128 itens: 65 itens na dimensão estrutura, 49 itens na dimensão processo e 14 itens na dimensão resultado. Seguidamente, a validação de conteúdo dos itens incluídos no instrumento foi concretizada mediante a análise de peritos (juízes) apresentada neste estudo.
Na fase de validação do conteúdo pelos peritos, inicialmente, para a apreciação de cada item do instrumento, numa escala tipo Likert com três pontos ${ }^{(18)}$ (discordo; nem discordo/nem concordo; concordo), foram considerados três critérios: a relevância, a clareza e a semelhança com outros itens. No que concerne à relevância, em cada item foi avaliada a pertinência para o construto e para a dimensão em que estava integrado. Além do parecer acerca dos critérios anteriormente mencionados, em relação a cada um dos itens, os peritos podiam expor outros comentários, de que são exemplo a necessidade de reformulação, o reposicionamento ou a exclusão imediata.

Os peritos foram selecionados de modo intencional, sendo: docentes, enfermeiros especialistas e enfermeiros gestores que se têm dedicado profissionalmente aos ambientes da prática profissional e à qualidade dos cuidados de enfermagem. A apresentação do estudo, o convite e o questionário eletrónico para participação foram enviados a 26 peritos, mas só foram devolvidas 22 respostas. Antes de avançarem para a resposta ao questionário, os peritos consentiram, de forma livre e esclarecida, a sua participação. Assim, e tal como recomendado pela literatura ${ }^{(18)}$, a amostra ficou constituída por 22 peritos, sendo 11 enfermeiros docentes e 11 enfermeiros em exercício profissional como enfermeiros especialistas ou enfermeiros gestores.

Embora a construção do instrumento tivesse início em janeiro de 2020, a coleta de dados junto dos peritos ocorreu durante os meses de abril e maio de 2020, por meio do questionário eletrónico, elaborado no Google Forms, contendo a caracterização dos peritos, bem como a proposta de avaliação de todos os itens do instrumento construído - a EAAPPE. No que se refere aos critérios avaliados, o item manteve-se quando, em relação à relevância e à clareza, a concordância dos peritos foi superior a $80 \%$. Sempre que os peritos apontassem semelhança com outros itens, o respetivo item era removido. Posteriormente, além do parecer dos peritos quanto aos três critérios mencionados, foi calculado o Índice de Validade de Conteúdo (IVC) ${ }^{(18-19)}$, que avalia a 
concordância dos peritos quanto à representatividade de cada item em relação ao conteúdo abordado. Se for $\geq 0,80$ traduz que o item é válido e deve ser mantido no instrumento ${ }^{(19)}$. Para o cálculo do IVC de cada item, dividiu-se o número de peritos que concordaram com o item pelo número total desses profissionais ${ }^{(18)}$.

Este estudo está integrado na investigação "Ambientes da Prática Hospitalar Promotores da Qualidade dos Cuidados de Enfermagem”, aprovada pelo comité de ética, com o número 137-20.

\section{Resultados}

Dos 22 peritos que participaram do estudo, embora todos fossem enfermeiros, 11 (50,0\%) eram docentes da área de enfermagem e 11 (50,0\%) exerciam funções como enfermeiros especialistas ou enfermeiros gestores. A maioria era do sexo feminino $(68,2 \%)$, casada ou a viver em união estável (90,9\%), com idade mínima de 32 anos e máxima de 64 anos, a idade média foi de 52,2 anos e desvio-padrão de 9,34. Com relação ao grau académico, 13,6\% eram licenciados, 36,4\% mestres e 50,0\% doutores. No que se refere à condição em que exerciam a profissão, 13,6\% eram professores coordenadores, $22,7 \%$ enfermeiros especialistas, 27,3\% enfermeiros gestores e 36,4\% professores adjuntos. O tempo de exercício profissional variou entre o mínimo de 10 anos e o máximo de 43 anos, com uma média de 30,3 anos e desvio-padrão de 9,46.

Tal como referido anteriormente, o instrumento submetido à validação dos peritos apresentava 128 itens distribuídos em três dimensões: estrutura, processo e resultado. Importa referir, com base no referencial teórico adotado ${ }^{(14)}$ e nos padrões de qualidade dos cuidados de enfermagem definidos em Portugal ${ }^{(20)}$, que a estrutura relaciona-se com os fatores organizacionais que permitem o desenvolvimento do trabalho dos profissionais de enfermagem, bem como as condições em que os cuidados são prestados; o processo compreende os fatores relacionados com o desempenho das atividades inerentes à concepção e prestação de cuidados de enfermagem, com base em padrões definidos; e o resultado consiste nas mudanças desejáveis ou indesejáveis, relativamente aos cuidados, aos clientes, bem como aos profissionais de enfermagem. Na sequência da avaliação efetuada pelos peritos, na dimensão estrutura, dos 65 itens propostos, 35 foram, na fase inicial, considerados adequados. Por outro lado, a existência de informações repetidas e a presença de aspectos vagos, difíceis de quantificar, determinaram a exclusão de 20 itens. As considerações dos peritos levaram à reformulação da redação de dez itens. O item relativo à disponibilização de serviços especializados para os enfermeiros que se deparam com situações problemáticas foi adicionado por sugestão dos peritos. Neste sentido, a segunda versão desta dimensão ficou constituída por 46 itens.

Para avaliar a pertinência de cada item quanto ao construto, foram calculados os IVC dos 46 itens cujos resultados estão apresentados no Quadro 1. Importa referir que todos os valores do IVC foram superiores a 0,80, traduzindo uma boa concordância entre os diferentes peritos quanto aos itens a incluir na dimensão Estrutura.

Quadro 1 - Concordância dos peritos em relação à representatividade quanto ao construto dos itens da dimensão Estrutura da Escala de Avaliação dos Ambientes da Prática Profissional de Enfermagem

(continua)

\begin{tabular}{|l|c|}
\hline Itens da estrutura & IVC $^{*}$ \\
\hline A instituição promove a participação dos enfermeiros na definição das políticas internas. & 0,95 \\
\hline $\begin{array}{l}\text { A instituição cria condições para que os enfermeiros possam agir em conformidade com os } \\
\text { objetivos definidos. }\end{array}$ & 0,91 \\
\hline Os enfermeiros conhecem o planeamento estratégico de enfermagem da instituição. & 0,86 \\
\hline $\begin{array}{l}\text { Na gestão de topo, os membros da direção de enfermagem têm poder semelhante aos } \\
\text { elementos das outras direções. }\end{array}$ & 0,91 \\
\hline $\begin{array}{l}\text { Os processos de comunicação entre os membros da gestão de topo, da gestão intermédia e } \\
\text { os profissionais são efetivos. }\end{array}$ & 0,91 \\
\hline
\end{tabular}


Quadro 1 - Concordância dos peritos em relação à representatividade quanto ao construto dos itens da dimensão Estrutura da Escala de Avaliação dos Ambientes da Prática Profissional de Enfermagem

(continuação)

\section{Itens da estrutura}

A política de formação institucional considera as necessidades formativas dos enfermeiros.

A instituição cria condições para que os enfermeiros possam investir na formação relevante para o seu desenvolvimento profissional.

A instituição reconhece a formação pós-graduada dos enfermeiros (especialidade, pós-graduação, mestrado, doutoramento).

A formação em serviço tem sido planeada com a colaboração dos enfermeiros.

IVC*

1,0

A instituição tem uma política de incentivo à inovação e à investigação em enfermagem.

A instituição promove a participação dos enfermeiros em comissões/grupos de trabalho no

âmbito da melhoria contínua da qualidade.

A instituição define indicadores de qualidade dos cuidados de enfermagem.

Na instituição, os projetos de melhoria contínua da qualidade levam em consideração os padrões de qualidade dos cuidados de enfermagem.

A instituição define uma cultura de segurança dos clientes.

A instituição define uma cultura de segurança dos enfermeiros.

O serviço apresenta um dimensionamento enfermeiro/clientes apropriado.

1,0

O serviço apresenta um dimensionamento enfermeiro especialista/clientes apropriado.

1,0

No serviço, trabalham enfermeiros com qualificações adequadas às necessidades dos clientes.

A instituição promove a mobilidade interna de enfermeiros entre serviços, de modo a colmatar défices de profissionais.

No serviço, a rotatividade de enfermeiros compromete a qualidade dos cuidados. 0,86

No serviço, adota-se um plano de integração para os enfermeiros recém-admitidos. 1,0

A instituição apresenta estratégias de motivação, bem como de recompensa e incentivos aos enfermeiros.

A instituição disponibiliza serviços especializados aos enfermeiros que se deparam com situações problemáticas.

Os equipamentos clínicos são adequados às necessidades do serviço.

As tecnologias de informação e comunicação são adequadas às necessidades do serviço. 0,91

A manutenção das infraestruturas do serviço é apropriada. 1,0

A manutenção dos equipamentos do serviço é apropriada. 1,0

O espaço disponível no serviço é adequado às necessidades dos clientes. 0,95

O ambiente físico é agradável e confortável para os enfermeiros. 0,91

Os enfermeiros são auscultados para a seleção de materiais e equipamentos.

No serviço, estão definidos os modelos teóricos de enfermagem que devem orientar a prática profissional dos enfermeiros.

Existe, no serviço, protocolos e procedimentos orientadores da prática de enfermagem.

No serviço, a distribuição dos clientes, pelos enfermeiros, é feita em função da intensidade, complexidade e continuidade dos cuidados.

A metodologia de trabalho de enfermagem adotada no serviço é promotora da qualidade

dos cuidados e garante práticas seguras.

O enfermeiro gestor orienta os enfermeiros numa atuação congruente com os padrões de

qualidade dos cuidados de enfermagem.

O enfermeiro gestor gere os conhecimentos e as capacidades de todos os enfermeiros da

equipa, de modo a que sejam alcançados os objetivos definidos.

O enfermeiro gestor utiliza os erros como oportunidades de aprendizagem. 
Quadro 1 - Concordância dos peritos em relação à representatividade quanto ao construto dos itens da dimensão Estrutura da Escala de Avaliação dos Ambientes da Prática Profissional de Enfermagem

(conclusão)

\begin{tabular}{|l|c|}
\hline Itens da estrutura & IVC* \\
\hline $\begin{array}{l}\text { O enfermeiro gestor apoia os enfermeiros da equipa nas dificuldades que emergem no dia } \\
\text { a dia, mesmo quando em conflito com outros profissionais. }\end{array}$ & 0,95 \\
\hline O enfermeiro gestor valoriza a opinião e as ideias inovadoras dos enfermeiros. & 1,0 \\
\hline Há equidade nos horários de trabalho e flexibilidade para alterações. & 1,0 \\
\hline O enfermeiro gestor proporciona momentos de reflexão sobre a prática. & 0,91 \\
\hline $\begin{array}{l}\text { O enfermeiro gestor cria condições que potenciam o desenvolvimento profissional dos } \\
\text { enfermeiros que lidera. }\end{array}$ & 1,0 \\
\hline $\begin{array}{l}\text { O enfermeiro gestor enaltece o empenho dos enfermeiros da equipa em melhorar } \\
\text { continuamente a qualidade dos cuidados. }\end{array}$ & 1,0 \\
\hline $\begin{array}{l}\text { Os enfermeiros têm oportunidade de participar na elaboração e implementação do plano } \\
\text { de ação do serviço. }\end{array}$ & 0,86 \\
\hline Há envolvimento e participação dos enfermeiros da equipa em processos de auditoria. & 0,91 \\
\hline $\begin{array}{l}\text { O enfermeiro gestor dá feedback aos enfermeiros sobre os indicadores, as auditorias e os } \\
\text { processos avaliativos da assistência de enfermagem. }\end{array}$ & 0,91 \\
\hline
\end{tabular}

Fonte: Elaboração própria.

*IVC: Índice de Validade de Conteúdo

Com relação à dimensão processo, na sequência da avaliação efetuada pelos peritos, dos 49 itens propostos, 39 foram, na fase inicial, considerados adequados. Por outro lado, a ausência de relevância do conteúdo e a existência de informações repetidas determinaram a exclusão de 8 itens. Perante as considerações dos peritos, foi reformulada a redação de dois itens. Neste sentido, a segunda versão da dimensão processo ficou constituída por 41 itens.

No que se refere à relevância de cada item quanto ao construto, os IVC estão apresentados no Quadro 2, importando referir que todos os valores foram superiores a 0,80, traduzindo uma boa concordância entre os diferentes peritos quanto aos itens a incluir no Processo.

Quadro 2 - Concordância dos peritos em relação à representatividade quanto ao construto dos itens da dimensão Processo da Escala de Avaliação dos Ambientes da Prática Profissional de Enfermagem

(continua)

\begin{tabular}{|l|c|}
\hline Itens do Processo & IVC* \\
\hline $\begin{array}{l}\text { A prática profissional dos enfermeiros está sustentada em referenciais teóricos de } \\
\text { enfermagem. }\end{array}$ & 0,86 \\
\hline $\begin{array}{l}\text { Os enfermeiros agem em consonância com os instrumentos reguladores do exercício } \\
\text { profissional. }\end{array}$ & 0,86 \\
\hline $\begin{array}{l}\text { Os enfermeiros empenham-se em sustentar a sua prática profissional na melhor evidência } \\
\text { científica. }\end{array}$ & 0,91 \\
\hline $\begin{array}{l}\text { Na prática profissional, os enfermeiros valorizam o conhecimento do domínio da } \\
\text { enfermagem. }\end{array}$ & 0,91 \\
\hline $\begin{array}{l}\text { A prática dos enfermeiros está fundamentalmente centrada na gestão de sinais e sintomas } \\
\text { da doença. }\end{array}$ & 0,95 \\
\hline $\begin{array}{l}\text { Os enfermeiros estão essencialmente focados na resposta às prescrições de outros } \\
\text { profissionais, numa valorização clara da dimensão interdependente. }\end{array}$ & 1,0 \\
\hline $\begin{array}{l}\text { Os enfermeiros têm a perceção de que, com a concretização das intervenções } \\
\text { interdependentes, o trabalho fica realizado. }\end{array}$ & 0,91 \\
\hline
\end{tabular}


Quadro 2 - Concordância dos peritos em relação à representatividade quanto ao construto dos itens da dimensão Processo da Escala de Avaliação dos Ambientes da Prática Profissional de Enfermagem

\begin{tabular}{|c|c|}
\hline Iten & IVC* \\
\hline $\begin{array}{l}\text { Tendencialmente, a prática dos enfermeiros é profundamente rotinizada, ao ponto de a } \\
\text { organização dos cuidados de enfermagem em cada turno ser determinada pelas rotinas. }\end{array}$ & 0,95 \\
\hline $\begin{array}{l}\text { Na prática dos enfermeiros, denota-se um enfoque significativo nas respostas humanas a } \\
\text { problemas reais e potenciais. }\end{array}$ & 0,91 \\
\hline Os enfermeiros evidenciam preocupação em valorizar as intervenções autónomas. & 0,95 \\
\hline A prática dos enfermeiros está fundamentalmente centrada na prevenção de complicações. & 0,91 \\
\hline $\begin{array}{l}\text { Os enfermeiros focam a sua atenção nas capacidades dos clientes, em detrimento de uma } \\
\text { perspetiva centrada na sua substituição. }\end{array}$ & 0,86 \\
\hline $\begin{array}{l}\text { Nos clientes com potencial, a prática dos enfermeiros está centrada na reconstrução da } \\
\text { autonomia. }\end{array}$ & 0,86 \\
\hline $\begin{array}{l}\text { Assistir os clientes nos processos de transição constitui o papel mais relevante dos } \\
\text { enfermeiros. }\end{array}$ & 0,95 \\
\hline $\begin{array}{l}\text { Os enfermeiros, na sua prática profissional, adotam modelos assistenciais centrados nos } \\
\text { clientes e, consequentemente, na individualização dos cuidados. }\end{array}$ & 0,91 \\
\hline $\begin{array}{l}\text { Os enfermeiros têm tempo para estar com os clientes e ir para além da resposta às } \\
\text { necessidades humanas básicas. }\end{array}$ & 0,86 \\
\hline Os enfermeiros demonstram autonomia na tomada de decisão sobre os cuidados. & 0,91 \\
\hline $\begin{array}{l}\text { Na avaliação inicial, os enfermeiros colhem, de forma rigorosa, os dados relevantes para a } \\
\text { concepção de cuidados de enfermagem. }\end{array}$ & 0,91 \\
\hline $\begin{array}{l}\text { Os diagnósticos de enfermagem traduzem as necessidades e os problemas dos clientes, } \\
\text { sejam eles pessoa doente, família ou cuidador. }\end{array}$ & 1,0 \\
\hline $\begin{array}{l}\text { Na concepção de cuidados, os enfermeiros centram-se nos clientes, mais do que no } \\
\text { processo de doença. }\end{array}$ & 0,95 \\
\hline Os enfermeiros promovem o envolvimento dos clientes no planeamento dos cuidados de enfermagem. & 0,91 \\
\hline Os enfermeiros avaliam os resultados das intervenções de enfermagem. & 0,91 \\
\hline Os planos de cuidados de todos os clientes são sistematicamente atualizados pelos enfermeiros. & 0,95 \\
\hline $\begin{array}{l}\text { Os enfermeiros documentam com exatidão, no sistema de informação em uso, os cuidados } \\
\text { planeados e executados. }\end{array}$ & 0,95 \\
\hline $\begin{array}{l}\text { A comunicação entre os membros da equipa é precisa e garante o planeamento adequado } \\
\text { dos cuidados. }\end{array}$ & 0,95 \\
\hline $\begin{array}{l}\text { O sistema de informação em suporte eletrónico dá resposta às necessidades de } \\
\text { documentação e contribui para a continuidade dos cuidados. }\end{array}$ & 0,91 \\
\hline $\begin{array}{l}\text { A informação transmitida na passagem de turno promove a continuidade dos cuidados nos } \\
\text { turnos subsequentes. }\end{array}$ & 1,0 \\
\hline A informação transmitida na passagem de turno é específica de enfermagem. & 0,95 \\
\hline $\begin{array}{l}\text { As estratégias adotadas para a passagem de turno, de que são exemplo a duração e o local, } \\
\text { são apropriadas para garantir a continuidade de cuidados. }\end{array}$ & 0,95 \\
\hline Existem relações de colaboração entre os diferentes membros da equipa de saúde. & 0,95 \\
\hline A relação de trabalho entre médicos e enfermeiros facilita a assistência aos clientes. & 0,95 \\
\hline A opinião clínica dos enfermeiros é considerada quando do planeamento da alta dos clientes. & 0,91 \\
\hline $\begin{array}{l}\text { O trabalho em equipa, que existe no serviço entre os enfermeiros, permite dar resposta às } \\
\text { necessidades de cuidados de enfermagem. }\end{array}$ & 0,91 \\
\hline $\begin{array}{l}\text { Existem, na equipa, momentos de partilha de conhecimento e de experiências sobre a } \\
\text { assistência aos clientes. }\end{array}$ & 0,95 \\
\hline $\begin{array}{l}\text { Quando da delegação de tarefas a profissionais funcionalmente dependentes, os } \\
\text { enfermeiros realizam uma supervisão apropriada. }\end{array}$ & 0,95 \\
\hline
\end{tabular}


Quadro 2 - Concordância dos peritos em relação à representatividade quanto ao construto dos itens da dimensão Processo da Escala de Avaliação dos Ambientes da Prática Profissional de Enfermagem

(conclusão)

\begin{tabular}{|l|c|}
\hline Itens do Processo & IVC* \\
\hline $\begin{array}{l}\text { No âmbito da equipa multiprofissional, há, entre os diferentes profissionais, compreensão e } \\
\text { valorização das respetivas funções e responsabilidades. }\end{array}$ & 0,95 \\
\hline A supervisão dos cuidados de enfermagem é uma atividade planeada e sistematizada. & 0,91 \\
\hline $\begin{array}{l}\text { A avaliação dos cuidados de enfermagem é realizada com base nos padrões de qualidade } \\
\text { dos cuidados de enfermagem. }\end{array}$ & 0,95 \\
\hline $\begin{array}{l}\text { Há reflexão sobre os indicadores de qualidade dos cuidados de enfermagem, de modo a } \\
\text { que sejam alcançados os objetivos definidos. }\end{array}$ & 0,95 \\
\hline $\begin{array}{l}\text { Há reflexão sobre as auditorias e os processos avaliativos da assistência de enfermagem, de } \\
\text { modo a promover a melhoria da qualidade dos cuidados. }\end{array}$ & 0,91 \\
\hline $\begin{array}{l}\text { As notificações de não conformidades são efetuadas como estratégia de melhoria contínua } \\
\text { da qualidade. }\end{array}$ & 0,91 \\
\hline
\end{tabular}

Fonte: Elaboração própria.

*IVC: Índice de Validade de Conteúdo

Com relação à dimensão resultado, na sequência da avaliação efetuada pelos peritos, dos 14 itens propostos, 10 foram, na fase inicial, considerados adequados. Por outro lado, a existência de informações repetidas determinaram a exclusão de 2 itens. Perante as considerações dos peritos, foi reformulada a redação de 2 itens e acrescentado o item relativo à avaliação de desempenho dos enfermeiros. Neste sentido, a segunda versão da dimensão resultado ficou constituída por 13 itens.

No que concerne à relevância de cada item quanto ao construto, os IVC estão apresentados no Quadro 3, observando-se que todos os valores foram superiores a 0,80, traduzindo uma boa concordância entre os diferentes peritos quanto aos itens a incluir na dimensão Resultado.

Quadro 3 - Concordância dos peritos em relação à representatividade quanto ao construto dos itens da dimensão Resultado da Escala de Avaliação dos Ambientes da Prática Profissional de Enfermagem

\begin{tabular}{|l|c|}
\hline Itens do Resultado & IVC* \\
\hline A cultura de segurança é monitorizada de forma sistemática. & 0,91 \\
\hline A qualidade dos cuidados de enfermagem é monitorizada de forma sistemática. & 0,95 \\
\hline A satisfação dos clientes com os cuidados prestados é monitorizada de forma sistemática. & 1,0 \\
\hline $\begin{array}{l}\text { Os indicadores de enfermagem são monitorizados, tendo em vista a melhoria contínua da } \\
\text { qualidade. }\end{array}$ & 0,91 \\
\hline Os indicadores de prevenção de complicações são monitorizados de forma sistemática. & 0,95 \\
\hline Os indicadores de ganhos em saúde são monitorizados de forma sistemática. & 0,95 \\
\hline $\begin{array}{l}\text { Os cuidados omissos (cuidados que ficam por realizar) são monitorizados de forma } \\
\text { sistemática. }\end{array}$ & 0,91 \\
\hline A satisfação profissional dos enfermeiros é monitorizada de forma sistemática. & 0,91 \\
\hline $\begin{array}{l}\text { A avaliação de desempenho dos enfermeiros é precisa e rigorosa, sendo reveladora do seu } \\
\text { real desempenho. }\end{array}$ & 0,95 \\
\hline O absentismo dos enfermeiros é monitorizado de forma sistemática. & 0,95 \\
\hline Os acidentes de trabalho dos enfermeiros são monitorizados de forma sistemática. & 0,86 \\
\hline A sobrecarga de trabalho dos enfermeiros é monitorizada de forma sistemática. & 0,86 \\
\hline A rotatividade de enfermeiros no serviço é monitorizada de forma sistemática. & 0,91 \\
\hline
\end{tabular}

Fonte: Elaboração própria.

*IVC: Índice de Validade de Conteúdo 


\section{Discussão}

Um ambiente da prática profissional de enfermagem favorável constitui um fator determinante para a qualidade da assistência, bem como para a obtenção de melhores resultados para os clientes, para os enfermeiros e para as instituições ${ }^{(6,10)}$. Embora no contexto internacional existam diversos instrumentos para avaliar os ambientes da prática $^{(6)}$, o desafio recai atualmente na necessidade de existirem instrumentos ajustados não só aos ambientes de trabalho contemporâneos, mas também ao desenvolvimento da Enfermagem e às particularidades do exercício profissional em diversos países. Nesse contexto, este estudo evidenciou-se importante, na medida em que permitiu proceder à validação de conteúdo de uma Escala de Avaliação dos Ambientes da Prática Profissional de Enfermagem. Nesse percurso de desenvolvimento do instrumento, cabe ressaltar a avaliação da relevância e clareza de cada item ter possibilitado aos peritos sugerirem modificações, o que aumentou a adequação desta ferramenta de análise aos contextos da prática.

A participação de 50,0\% de peritos da área académica e 50,0\% da área clínica contribuiu para aumentar a robustez da atual versão do instrumento, na medida em que estão contemplados os aspectos mais majorados, quer na componente teórica, quer na componente prática. Esta opção metodológica permitiu selecionar, de forma rigorosa, os itens que representam as dimensões a serem consideradas nos ambientes da prática profissional de enfermagem favoráveis à qualidade dos cuidados.

Numa revisão da literatura, os autores confirmaram que os domínios mapeados nos diversos instrumentos para avaliação dos ambientes da prática profissional de enfermagem, apesar de evidenciarem pouca consistência na terminologia, são sobrepostos, tendo sido destacados: a liderança, o trabalho em equipa, a carga de trabalho, a autonomia, a participação, o relacionamento com os pacientes, o desenvolvimento profissional, os recursos estruturais e eletrónicos, o salário e os benefícios e a cultura de segurança ${ }^{(6)}$.
No caso do instrumento construído em consonância com o referencial de Donabedian, a dimensão estrutura deve contemplar recursos organizacionais que permitam o desenvolvimento do trabalho dos enfermeiros, bem como fatores relativos às condições em que os cuidados são $\operatorname{prestados}^{(14)}$. Assim, ainda que não estejam definidos à priori os fatores incluídos em cada umas das dimensões do instrumento proposto neste estudo, o que só acontecerá nas fases seguintes de validação, os 46 itens incluídos na versão atual da dimensão estrutura reportam-se a fatores organizacionais, fatores relacionados com a formação, inovação e investigação em enfermagem, fatores relativos à qualidade e segurança dos cuidados, fatores referentes à gestão de pessoas e de recursos materiais, fatores relacionados com a organização e sustentabilidade da prática de enfermagem, bem como fatores relativos à gestão e liderança no serviço.

Em consonância com a literatura, os itens incluídos na estrutura reforçam a necessidade de proporcionarem-se aos enfermeiros apropriadas condições de trabalho, de desenvolvimento profissional e de participação na instituição ${ }^{(3,5)}$; de assegurar-se a dotação de enfermeiros em quantidade e qualidade adequadas ${ }^{(21)}$; de investir-se em metodologias de organização dos cuidados de enfermagem que, ao diminuírem a carga de trabalho dos enfermeiros, permitam dar resposta às necessidades dos pacientes, reduzindo o risco de eventos adversos e impedindo a deterioração da condição clínica ${ }^{(22)}$ e, simultaneamente, de se garantir nos serviços uma gestão/liderança capaz de fazer a diferença. Estudos evidenciam que os enfermeiros gestores têm impacto direto no desempenho e bem-estar dos enfermeiros das equipes que lideram, bem como na qualidade dos cuidados prestados ${ }^{(5,9,23)}$. Nos esforços que os gestores devem realizar para melhorar os ambientes da prática, é crucial reconhecer e atender às necessidades dos profissionais de enfermagem $^{(3)}$. Entretanto, a par das estratégias de motivação e suporte ${ }^{(24)}$, a equidade nos horários de trabalho e a flexibilidade para alterações ${ }^{(25)}$ são também fundamentais na promoção do envolvimento desses profissionais. 
Novamente em consonância com o referencial de Donabedian, na dimensão processo, o enfoque está nos fatores relacionados com o desempenho das atividades inerentes à concepção e prestação de cuidados de enfermagem ${ }^{(14)}$. Nesse contexto, e ainda que não estejam definidos a priori, os 41 itens incluídos na versão atual reportam-se a fatores relacionados com o desenvolvimento da prática profissional, fatores relativos aos modelos assistenciais, fatores referentes à metodologia científica adotada na prestação de cuidados, fatores relativos ao processo de comunicação e à continuidade de cuidados, fatores relacionados às práticas colaborativas e relações multiprofissionais e fatores referentes aos processos de supervisão e avaliação dos cuidados de enfermagem.

Importa destacar que os itens incluídos na dimensão processo advertem, mais uma vez, que a atuação do enfermeiro deve estar sustentada nos referenciais teóricos da disciplina e nos instrumentos que regulam o exercício profissional ${ }^{(7)}$. Além disso, potenciará a adoção de modelos assistenciais centrados nas pessoas e nas transições que vivenciam, ao invés de focados na patologia, o que culminará numa adequada valorização da dimensão autónoma do exercício profissional ${ }^{(26)}$.

Por último, ainda em consonância com o referencial de Donabedian, na dimensão resultado, o enfoque está nas mudanças desejáveis ou indesejáveis, relativamente à instituição, aos cuidados, aos clientes, bem como aos profissionais ${ }^{(14)}$. Assim, no que se refere a esta dimensão, os 13 itens reportam-se à relevância de monitorizar os resultados relativos à instituição, aos cuidados e clientes e aos enfermeiros. Conforme descrito na literatura, um ambiente de prática profissional de enfermagem favorável caracteriza-se por maior satisfação profissional, melhor desempenho, maior qualidade da assistência prestada e menores níveis de absenteísmo o que, consequentemente, melhora a eficiência, a viabilidade financeira e o clima de segurança institucional ${ }^{(5,9)}$, bem como a experiência vivenciada pelos clientes e pelos próprios profissionais. Neste contexto, nenhum ambiente da prática profissional de enfermagem pode ser integralmente favorável, se não existir evidência da preocupação com a monitorização dos potenciais resultados.

Quanto aos valores obtidos no IVC, ainda que a EAAPPE apresente adequada validade de conteúdo, importa salientar que este estudo corresponde apenas a uma das primeiras fases da construção de instrumentos, fazendo-se necessária a sua aplicação à população alvo, com posterior realização de procedimentos psicométricos, o que já se encontra em andamento em estudo multicêntrico. Ainda que a Escala tenha sido construída com base na prática dos enfermeiros, a validação pelos peritos foi essencial, uma vez que, além de confirmarem a relevância dos itens, foram determinantes na melhoria da redação e, consequentemente, na adequação do instrumento.

Em síntese, os 100 itens incluídos na atual versão da EAAPPE contemplam aspectos relacionados com a estrutura, o processo e o resultado, reforçando a relevância de serem considerados todos os fatores nos ambientes da prática profissional de enfermagem promotores da qualidade dos cuidados. Tal como defendem alguns autores, a aplicação de um instrumento com essas particularidades será fundamental para que os gestores conheçam as características dos ambientes, fornecendo simultaneamente subsídios para a adoção das estratégias que melhor os qualifiquem $^{(27)}$.

Assume-se, no entanto, como limitação, o fato de os peritos não serem de todas as regiões do país.

\section{Conclusão}

A validação de conteúdo da Escala de Avaliação dos Ambientes da Prática Profissional de Enfermagem permitiu confirmar a relevância teórica de cada um dos itens incluídos nas três dimensões que a constituem. O percurso efetuado até ao momento indica que o instrumento reflete o propósito para o qual será usado. Assim, em próximos estudos, sugere-se dar continuidade ao processo de elaboração do instrumento, nomeadamente a procedimentos exigidos para a sua validação.

Sustentado no referencial teórico de Donabedian, no futuro, a utilização desta métrica 
12

Escala de avaliação dos ambientes da prática profissional de enfermagem: construção e validação de conteúdo

permitirá avaliar os componentes estrutura, processo e resultado dos ambientes da prática profissional de enfermagem, configurando-se como uma ferramenta útil à definição de estratégias que garantam ambientes favoráveis à qualidade dos cuidados de enfermagem.

\section{Colaborações:}

1 - concepção, projeto, análise e interpretação dos dados: Olga Maria Pimenta Lopes Ribeiro, Corália Maria Fortuna de Brito Vicente, Maria Manuela Ferreira Pereira da Silva Martins e Letícia de Lima Trindade;

2 - redação do artigo e revisão crítica relevante do conteúdo intelectual: Olga Maria Pimenta Lopes Ribeiro, Letícia de Lima Trindade, Clemente Neves de Souza e Maria Filomena Passos Teixeira Cardoso;

3 - aprovação final da versão a ser publicada: Olga Maria Pimenta Lopes Ribeiro, Corália Maria Fortuna de Brito Vicente, Maria Manuela Ferreira Pereira da Silva Martins, Letícia de Lima Trindade, Clemente Neves de Souza e Maria Filomena Passos Teixeira Cardoso.

\section{Referências}

1. Jones A, Whyley H, Doyle J, Bevan L. Development of approaches and legislation to optimise nurse staffing levels. Nurs Stand. 2018;33(5):27-31. DOI: 10.7748/ns.2018.e11202

2. Stimpfel AW, Djukic M, Brewer CS, Kovner CT. Common predictors of nurse-reported quality of care and patient safety. Health care management review. Health Care Manage Rev. 2019;44(1):57-66. DOI: 10.1097/HMR.0000000000000155

3. Santos JLG, Erdmann AL, Peiter CC, Alves MP, Lima SBS, Backes VMS. Comparação entre ambiente de trabalho de enfermeiros gerentes e assistenciais no contexto hospitalar. Rev Esc Enferm USP. 2017;51:e03300. DOI: 10.1590/ s1980-220x2017017103300

4. Lake E. Development of the Practice Environment Scale of the Nursing Work Index. Res Nurs Health. 2002;25(3):176-88. DOI: 10.1002/nur.10032

5. Wei H, Sewell KA, Woody G, Rose MA. The state of the science of nurse work environments in the
United States: A systematic review. Int J Nurs Sci. 2018;5(3):287-300. DOI: 10.1016/j.jinss.2018.04.010

6. Norman RM, Sjetne IS. Measuring nurses' perception of work environment: a scoping review of questionnaires. BMC Nurs. 2017;16(1):1-15. DOI: $10.1186 / s 12912-017-0256-9$

7. Ribeiro OM, Martins MM, Tronchin DM, Silva JM. Professional nursing practice based on theoretical framework of the discipline: is it reality or utopia. Rev Enf Ref. 2018;IV(19):39-48. DOI: 10.12707/ RIV18040

8. Jesus E, Roque S, Amaral A. Estudo RN4CAST em Portugal: Ambientes de Prática de Enfermagem. Rev Investig Enferm [Internet]. 2015 [cited 2020 May 30];13:26-44. Available from: https://docplayer. com.br/65068922-Estudo-rn4cast-em-portugalambientes-de-pratica-de-enfermagem.html

9. Chen J, Ramalhal T, Lucas PB. Nursing Practice Environment and Nurses' Satisfaction in a Hospital Context - A Scoping Review. Pensar Enfermagem [Internet]. 2019 [cited 2020 May 31];23(2):29-42. Available from: http://pensarenfermagem.esel.pt/ files/PE_2SEM2019_miolo_final-29-42.pdf

10. Copanitsanou P, Fotos N, Brokalaki H. Effects of work environment on patient and nurse outcomes. Br J Nurs. 2017;26(3):172-6. DOI: 10.12968/ bjon.2017.26.3.172

11. World Health Organization. Fact Sheet - WHO and partners call for urgent investment in nurses [Internet]. Geneva; 2020 [cited 2020 May 16]. Available from: https://www.icn.ch/news/whoand-partners-call-urgent-investment-nurses?fbclid =IwAR2gRxgs6OMnMoqHZAF7IkrT6xZOsfsd5HP gpgOwbK3wFksYZBzY7PFRjeo

12. Aiken LH, Patrician PA. Measuring Organizational Traits of Hospitals: The Revised Nursing Work Index. Nurs Res. 2000;49(3):146-53. DOI: 10.1097/00006199-200005000-00006

13. Ribeiro OMPL, Vicente CMFB, Martins MMFPS, Vandresen L, Silva JMAV. Instruments for assessing professional nursing practice environments: an integrative review. Rev Gaúcha Enferm. 2020;41:e20190381. DOI: 10.1590/1983-1447.2020.20190381

14. Donabedian A. An Introduction to Quality Assurance in Health Care. New York: Oxford University Press; 2003.

15. Ferreira MR, Amendoeira J. Study of adaptation and validation of the Practice Environment Scale of the Nursing Work Index for the Portuguese 
Reality. Rev Esc Enferm USP. 2014;48(8):690-7. DOI: $10.1590 /$ s0080-623420140000400017

16. Amaral A, Ferreira L, Lake E. Validation of the Practice Environment Scale of the Nursing Work Index (PES-NWI) for the Portuguese nurse population. Int J Caring Sci [Internet]. 2012 [cited 2020 Aug 16];5(3):280-8. Available from: https: //doaj.org/article/1ec29cd2c2c14bed8bc3b5f 3c4464b0a

17. Ribeiro OM. Conceções e Práticas dos Enfermeiros. Olhares sobre um percurso em hospitais portugueses. Loures: Lusodidacta; 2018.

18. Medeiros RKS, Ferreira Junior MA, Pinto DPS, Vitor AF, Santos VEP, Barichello E. Pasquali's model of content validation in the Nursing researches. Rev Enf Ref. 2015;IV(4):127-35. DOI: 10.12707/RIV14009

19. Souza AC, Alexandre NM, Guirardello ED. Psychometric properties in instruments evaluation of reliability and validity. Epidemiol Serv Saude. 2017;26(3):649-59. DOI: 10.5123/ s1679-49742017000300022

20. Ordem dos Enfermeiros. Padrões de qualidade dos cuidados de enfermagem [Internet]. Lisboa; 2012 [cited 2020 Aug 15]. Available from: https:// www.ordemenfermeiros.pt/media/8903/divulgarpadroes-de-qualidade-dos-cuidados.pdf

21. Bettencourt AP, McHugh MD, Sloane DM, Aiken LH. Nurse Staffing, the Clinical Work Environment, and Burn Patient Mortality. J Burn Care Res. 2020;41(4):796-802. DOI: 10.1093/jbcr/ iraa061

22. Fagerström L, Kinnunen M, Saarela J. Nursing workload, patient safety incidents and mortality: an observational study from Finland. BMJ Open. 2018;8(4):e016367. DOI: 10.1136/ bmjopen-2017-016367

23. Bogaert PV, Peremans L, Heusden DV, Verspuy M, Kureckova V, Cruys ZVD, et al. Predictors of burnout, work engagement and nurse reported job outcomes and quality of care: a mixed method study. BMC Nurs. 2017;16(5):1-14. DOI: $10.1186 /$ s12912-016-0200-4

24. Wan Q, Zhou W, Li Z, Shang S, Yu F. Work engagement and its predictors in registered nurses: A cross-sectional design. Nurs Health Sci. 2018;20(4):415-21. DOI: 10.1111/nhs.12424

25. Dhaini SR, Denhaerynck K, Bachnick S, Schwendimann R, Schubert M, Geest SD, et al. Work schedule flexibility is associated with emotional exhaustion among registered nurses in Swiss hospitals: A cross-sectional study. Int J Nurs Stud. 2018;82:99-105. DOI: 10.1016/j. ijnurstu.2018.03.019

26. Ribeiro OMPL, Martins MMFPS, Tronchin DMR, Silva JMAV, Forte ECN. Professional practice models used by nurses in Portuguese hospitals. Rev Bras Enferm. 2019;72(suppl 1):24-31. DOI: 10.1590/0034-7167-2017-0670

27. Gasparino RC, Ferreira TDM, Carvalho KMA, Rodrigues ESA, Tondo JCA, Silva VA. Evaluation of the professional practice environment of nursing in health institutions. Acta paul enferm. 2019;32(4):449-55. DOI: 10.1590/19820194201900061

Recebido: 21 de julho de 2020 Aprovado: 20 de agosto de 2020 Publicado: 14 de outubro de 2020

A Revista Baiana de Enfermagem utiliza a Licença Creative Commons - Atribuição-NãoComercial 4.0 Internacional. https://creativecommons.org/licenses/by-nc/4.0/

Este artigo é de acesso aberto distribuído sob os termos da Licença Creative Commons (CC BY-NC). Esta licença permite que outros remixem, adaptem e criem a partir do seu trabalho para fins não comerciais. Embora os novos trabalhos tenham de lhe atribuir o devido crédito e não possam ser usados para fins comerciais, os usuários não têm de licenciar esses trabalhos derivados sob os mesmos termos. 